\title{
Spiraling and Folding: The Word View
}

\author{
Marcus Schaefer • Eric Sedgwick • Daniel Štefankovič
}

\begin{abstract}
We show that for every $n$ there are two simple curves on the torus intersecting at least $n$ times without the two curves folding or spiraling with respect to each other. On the other hand, two simple curves in a punctured plane that intersect at least $n$ times (and do not create any empty bigons) must either form a spiral of depth $d$ or a fold of width $c n /(d+1)-1$, where $c$ only depends on the number of punctures in the plane. The construction of the two curves on the torus involves train tracks and word equations, and the verification that the two curves do not spiral leads us to an infinite binary word based on the golden ratio which does not contain any square word $w w$ for which $|w|$ is even.
\end{abstract}

Keywords Curves · Surfaces · Topology $\cdot$ Square-free words $\cdot$ Thue word · Spirals · Folds

\section{Introduction}

Maybe you have found yourself aimlessly doodling away on a piece of paper, producing psychedelic drawings like 


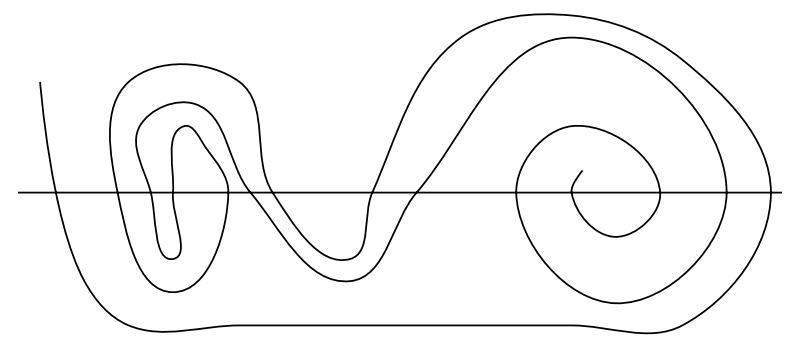

As you are squeezing one more bend of the curve into the picture you might have wondered whether the doodle has any inherent structure. The picture above, for example, contains a fair amount of spiraling and folding. If your curves intersect often, do they always have to spiral or fold? And does this depend on the surface on which you are drawing?

Before we discuss these questions, we need to make our notions of folding and spiraling precise. We are interested in the behavior of two simple curves, that is, curves without self-intersections. We typically draw one of the curves as a straight line; it might be part of a triangulation of the surface. The second curve intersects the first curve a large number of times. Two curves are reduced (with respect to each other) if their drawing does not contain a lens: a lens (or empty bigon), is a discshaped region bounded by two arcs, one from each curve, that does not contain any other part of the curve in its interior. Requiring the curves to be reduced seems to seriously restrict doodling, but we can always eliminate a lens by punching a hole into the lens, puncturing the surface. The earlier picture contains four lenses which could be removed by adding four punctures to the surface. ${ }^{1}$

An annulus is a disk with one puncture. An $\operatorname{arc}$ in $A$ is a curve within $A$ with endpoints on the boundary of $A$. If the endpoints are on both boundary components, we call the arc spanning, otherwise it is peripheral. Two spanning arcs in an annulus $A$ are said to spiral if they do not form a lens within $A$ and intersect at least three times. The number of intersections minus 2 is the depth of the spiral. Two curves $\alpha$ and $\beta$ spiral if there is an annulus $A$ and two subarcs $\alpha^{\prime} \subseteq \alpha$ and $\beta^{\prime} \subseteq \beta$ such that $\alpha^{\prime}$ and $\beta^{\prime}$ spiral in $A$ (note that $\alpha^{\prime}$ and $\beta^{\prime}$ are spanning arcs in $A$ ).

We say that two curves $\alpha$ and $\beta$ have a fold of width $w$ if there is an annulus $A$ that does not contain endpoints of either $\alpha$ or $\beta$ and so that $\alpha$ intersects $A$ in a peripheral arc $\alpha^{\prime}$, and the intersection of $\beta$ and $A$ contains at least $w$ peripheral arcs each of which intersects $\alpha^{\prime}$ twice without forming a lens with $\alpha^{\prime}$. We require a fold to have width at least 1 .

The following picture shows that our opening example contained a fold of width three and a spiral of depth one (the two annuli are crosshatched).

\footnotetext{
${ }^{1}$ Topologically speaking: lenses are topologically "trivial", since they can be removed by an isotopy of either curve. Of course any curve on the plane can be isotoped into a point, so if we take the more topological view-instead of speaking of reduced curves - we would need to anchor the four endpoints of the curves by placing them on the boundaries of four punctures in the plane.
} 


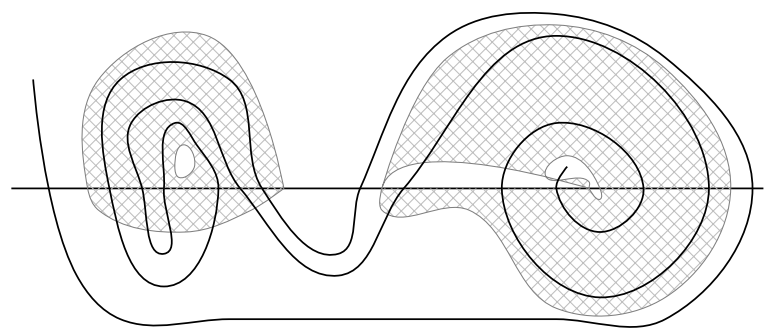

The following example shows that in spite of an arbitrarily large number of intersections of $\alpha$ and $\beta$ there need not be any spiraling and only very narrow folding in a drawing:

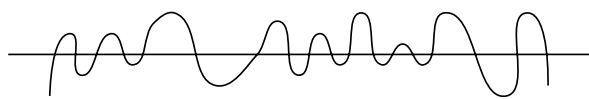

However, for this to be possible with reduced curves, the plane would need to contain a large number of punctures, so let us assume that we are dealing with a fixed surface, that is, the number of punctures is fixed.

Does a large number of intersections between two reduced curves on a fixed surface force either a deep spiral or a wide fold?

We will show in Sect. 2 that in the punctured plane there is a constant $c=O(p)$ depending only on the number $p$ of punctures such that any reduced curves with $n$ intersections contain either a spiral of depth $\sqrt{n} / c$ or a fold of width $\sqrt{n} / c$. In Sect. 3 we construct two reduced curves on the torus with arbitrary many intersections which form neither spiral nor fold.

The question above is not an entirely idle problem inspired by doodling on scraps of paper, it is closely related to graph drawing problems, and the string graph problem in particular. It is easy to show, adapting a construction due to Kratochvíl and Matoušek [8] for string graphs, that in the presence of $n$ punctures two curves can be forced to intersect on the order of $\Omega\left(2^{n}\right)$ times in the plane without there being a spiral. These curves do, however, contain a fold of width $\Omega\left(2^{n}\right)$. In the companion paper to this paper we construct two reduced curves in the (unpunctured) plane without any spirals and with arbitrarily many intersections [16]. By the result mentioned earlier, these two curves have a wide fold.

In the present paper we construct curves $\alpha$ and $\beta$ on the torus that form no spiral and no fold. This closes a promising approach to recognizing string graphs on surfaces of higher genus. A string graph is the intersection graph of simple curves in the plane (or another surface). Recognizing whether a graph is a string graph is an old question $[2,6]$ that was settled only recently by proving an exponential upper bound on the number of intersections needed in a realization of a string graph in the plane $[11,12]$.

There are three known approaches to proving the decidability of string graphs in the plane: the topological proof by Pach and Tóth [11], our more combinatorial proof from [12] and an algebraic proof using trace monoids [14]. The last proof is the only one that currently works for arbitrary surfaces. Unfortunately, it only gives a doubleexponential upper bound on the number of intersections in an optimal realization of a string graph on a surface. The proof from [12] makes essential use of properties of 
the plane, and does not seem to lift easily to other surfaces. This leaves the proof of Pach and Tóth which proceeds by finding a deep spiral in the realization of a string graph which then allows the simplification of that realization. Our main result of this paper implies that this approach cannot be lifted in a straightforward manner even to the torus, since there are pairs of curves that do not form any spirals.

The companion paper [16] establishes Theorem 3.1 - that folds and spirals can be avoided on the torus - using entirely topological methods; our proof of this result in the present paper follows a different approach: we capture the behavior of curves in surfaces using words (over monoids) and word equations. To this end, we represent curves on the torus using Thurston's train tracks. Relevant properties of curves can then be described using word equations: We construct a family of curves using train tracks and show that they do not form a spiral by proving that the solution of a word equation associated with the train track does not contain any squares, that is words of the form $w w .^{2}$

The word view has an advantage over the topological approach in that it lends itself more naturally to automation: the solutions to the word equations we derive can easily be computed and verified, and basic properties of curves represented by train tracks can be decided efficiently, following ideas from [13].

Also, the word view leads us to new results about square-free words; during the proof we encounter an infinite binary word which does not contain any squares of the form $w w$ where $|w|$ is even. Interestingly, the word has both a natural definitionits $n$th digit is $\lfloor n \phi \bmod 2\rfloor$, where $\phi$ is the golden ratio-and a simple recursive construction which connects it to the word equations we use to encode our train tracks.

Remark 1.1 Given an infinite binary word a without squares of the form $w w$ with $|w|$ even, we can combine it with the infinite word $\mathbf{b}=01010101 \ldots$ which does not contain any squares $w w$ with $|w|$ odd to build an infinite word on a 4-letter alphabet that does not contain any squares $w w$ at all (one letter corresponding to each combination $00,01,10$, and 11 ). Thue showed that square-free words exist over a ternary alphabet [9], but as far as we know all known constructions involve morphisms of words and are not direct (though there are some recursive constructions).

If we interleave the letters of $\mathbf{a}$ and $\mathbf{b}$, we obtain an infinite binary word which does not contain any squares $w w$ with $|w|>3$. In particular, the interleaved word contains only a finite number of different squares; this is an old result, again typically proved through the use of morphisms. The best result known is that there are words that contain only three different squares, 00, 11, 0101 [5].

\section{The Planar Case}

Spirals and folds can be avoided entirely on the torus as we will see in the next section. In the plane with a fixed number of punctures, however, there always has to

\footnotetext{
${ }^{2}$ Studying curves in surfaces and their properties using words and word equations is an approach we first used in our papers on string graphs [12,14] and continued with papers on algorithms for curves represented by Thurston train tracks or through normal coordinates [13, 15].
} 
be a spiral or fold of size $\Omega\left(\ell^{1 / 2}\right)$, where $\ell$ is the number of intersections between two reduced curves.

Theorem 2.1 Two reduced curves intersecting $\ell$ times in a plane with $p$ punctures form either a spiral of depth $d$ or a fold of width $\ell / c_{p} d-1$, where $c_{p}=2(12 p+13)^{2}$.

A similar result is implicit in the paper by Pach and Tóth [11] and some of our arguments resemble theirs. We obtain a slightly better constant $c_{p}$ by using a genus argument.

Consider two reduced curves $\alpha$ and $\beta$ which intersect a finite number of times. Removing the two curves from the plane decomposes the plane into a number of regions we call cells. A segment of a curve is a connected component of $\alpha \backslash \beta$ and $\beta \backslash \alpha$. We call a segment proper if it does not contain an endpoint of the curve; the two segments of a curve that are not proper are called its end segments. A $k$-cell is a cell whose boundary, after erasing the end segments of $\alpha$ and $\beta$, consists of $k$ proper segments. A cell is good if it is an empty 4-cell, that is, it does not contain any punctures or endpoints, and it is bad otherwise.

Arbitrarily orient $\alpha$ in one direction. Call a segment of $\alpha \operatorname{good}$ if it is proper and its right side borders a good cell, otherwise the segment is bad. Our first goal is to bound the number of bad segments.

Lemma 2.2 In a drawing of two reduced curves in a plane with punctures a curve has at most $12 p+12$ bad segments not counting the two end segments of the curve.

Proof Fix a drawing of two reduced curves $\alpha$ and $\beta$. Consider the dual multigraph $G$ of the drawing: assign a vertex to each cell and connect two vertices by an edge if their cells share a proper segment. Note that $G$ may contain multiple edges, but no loops.

Erase the four end segments from the drawing. Then the drawing has $\ell$ vertices, namely the $\ell$ intersection points of the curves, and $2(\ell-1)$ edges (the proper segments), and hence, by Euler's formula there are $2+2(\ell-1)-\ell=\ell$ faces, implying that $G$ has $\ell$ vertices. Moreover, the number of edges of $G$ is $2(\ell-1)$, since an edge in $G$ corresponds to a proper segment of the drawing. Vertices of degree $k$ in $G$ correspond to $k$-cells in the drawing. In particular, $G$ contains at most $p$ vertices of degree 2 , since each 2 -cell must contain a puncture (otherwise it would be a lens) and at most 4 vertices of degree 3 , since a 3-cell must contain one of the four endpoints. Let $d_{k}$ be the number of $k$-cells and let $d_{4}^{b}$ and $d_{4}^{g}$ be the number of bad and good 4-cells, resp., so $d_{4}=d_{4}^{b}+d_{4}^{g}$.

Assuming that $\ell \geq 2, G$ contains no isolated vertices and we get

$$
2 d_{2}+3 d_{3}+4 d_{4}+5\left(\ell-d_{4}-d_{3}-d_{2}\right) \leq 2|E(G)|=4(\ell-1) .
$$

Replacing $d_{4}$ by $d_{4}^{b}+d_{4}^{g}$ gives us $\ell-d_{4}^{g} \leq 3 d_{2}+2 d_{3}+d_{4}^{b}-4$. Every 2 -cell contains a puncture, every 3-cell an endpoint, and every bad 4-cell an endpoint or a puncture, so $3 d_{2}+2 d_{3}+d_{4}^{b}=2 d_{2}+d_{3}+\left(d_{2}+d_{3}+d_{4}^{b}\right) \leq 2 p+4+(p+4)=3 p+8$. This implies that $\ell-d_{4}^{g} \leq 3 p+4$. 
Now the bad cells border at most $2|E(G)|-4 d_{4}^{g}$ segments, and

$$
2|E(G)|-4 d_{4}^{g}=4\left(\ell-d_{4}^{g}\right)-4 \leq 12 p+12,
$$

so there are at most $12 p+12$ bad segments not counting the end segments of the two curves.

Proof of Theorem 2.1 Fix a drawing of reduced curves $\alpha$ and $\beta$ and a direction of travel along $\alpha$. Label the segments encountered $\alpha_{0}, \ldots, \alpha_{\ell}$. We write $\alpha[i: j]$ for the sequence $\alpha_{i}, \ldots, \alpha_{j}$. Each segment is adjacent to a cell on its left and its right (not all these cells are pairwise distinct, of course). Let us consider the $\ell-1$ cells $\alpha[1: \ell-1]$ encountered along the right side of $\alpha$ excluding the two end segments. Since at most $12 p+12$ of these segments are bad, there is a sequence of at least $w=\lceil(\ell-1-$ $(12 p+12)) /((12 p+12)+1)\rceil \geq \ell /(12 p+13)-1$ consecutive segments, $\alpha[i: i+$ $w-1]$, such that the cells on the right of the segments are all empty 4-cells. This block of 4-cells attaches to $\alpha$ a second time, again to a block of segments, $\alpha[j: j+$ $w-1]$; the rest of the argument depends on where and how it does this. Figure 1 illustrates one possible scenario, in which the cells reattach to the opposite side of $\alpha$, overlapping the original block.

If the block of 4-cells attaches on the same side of $\alpha$, we have found a fold of width $w$ and we are done since $w \geq \ell /(12 p+13)-1 \geq \ell / c_{p} d-1$. Hence, we can assume that the block of 4-cells attaches to the left side of $\alpha$.

If the two blocks $\alpha[i: i+w-1]$ and $\alpha[j: j+w-1]$ overlap in more than $w(d-1) / d$ segments, we have found a spiral of depth $d$ : in case $i<j$ start with $\alpha_{j}$ and follow the empty 4-cell on its right. It reattaches to $\alpha$ in $\alpha_{j+(j-i)}$; continuing this process, we obtain a sequence of empty 4-cells connecting $\alpha_{i+k(j-i)}$ to $\alpha_{i+(k+1)(j-i)}$ for $0 \leq k \leq d$. This is possible since all those cells start within the range $\alpha[i: i+w-1]$ as $j-i<w-w(d-1) / d=w / d$ and so $i+d(j-i) \leq i+w-1$. Pick one of the two subarcs of $\beta$ bounding these 4-cells. It has $d+2$ intersections with $\alpha$, so we have found a spiral of depth $d$. The case $j<i$ is symmetric.

We can therefore assume that the two blocks overlap in at most $w(d-1) / d$ segments; then there are at least $w / d$ consecutive segments $\alpha\left[j^{\prime}: j+w-1\right], j \leq j^{\prime}$ that are not involved in the overlap and that have good 4-cells on their left side. Let $\alpha^{\prime}$ be the subarc $\alpha[i+w: j+w-1]$ (this includes $\alpha\left[j^{\prime}: j+w-1\right]$ since these segments are not part of the overlap). Note that $\alpha^{\prime}$ together with the segment of $\beta$ connecting the beginning of $\alpha[i+w]$ to the end of $\alpha[j+w-1]$ forms a closed curve $C$.

Temporarily replace $\alpha$ with $\alpha^{\prime}$ (without changing $\beta$ ). Now $\alpha^{\prime}$ has at most $12 p+12$ bad segments (we can apply Lemma 2.2 again), so it contains a block $\alpha\left[k: k+w^{\prime}-1\right]$

Fig. 1 A block of 4-cells $\alpha[i: i+w-1]$ reattaching to $\alpha$ on the opposite side in $\alpha[j: j+w-1]$ overlapping the original block $\alpha[i: i+w-1]$ in $\alpha[j: i+w-1]$

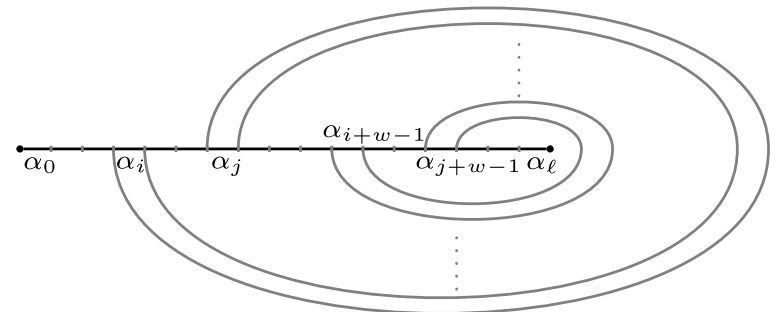


of good segments, where $w^{\prime}=\lceil w /(d(12 p+13))\rceil-1$. Since the cells on the right of these segments are all contained within the closed curve $C$, the block of cells has to reattach to $\alpha^{\prime}$ on the right hand side, so in $\alpha^{\prime}$ we now have a wide fold. In general, this fold will not be a fold with respect to $\alpha$, since $\alpha$ can cut through the cells of the fold. But if we look at the cells of the fold between $\alpha^{\prime}$ and $\beta$ in the presence of $\alpha$, we see that all the cells remain good, with one possible exception: the cell that contains the endpoint of $\alpha$ in the region bounded by $C$. Let us pick a block of cells on the right of the interval $\alpha\left[k: k+w^{\prime}-1\right]$. If all the cells are good, this block has to reattach on the right or the left side of $\alpha$ as a block. We can then continue on the other side of that block, as long as all those cells are good as well. If we keep encountering good cells, this process will continue until we reattach to $\alpha$ on the right side (we know we must, since we are following the fold with respect to $\alpha^{\prime}$ ). We saw that there is at most one bad cell we can encounter, so either $\alpha\left[k: k+\left\lfloor w^{\prime} / 2\right\rfloor-1\right]$ or $\alpha\left[k+\left\lfloor w^{\prime} / 2\right\rfloor: k+w^{\prime}-1\right]$ will not run into that bad cell. Since the process starts on the right-hand side of $\alpha$ and ends on the right-hand side of $\alpha$, there must be a block of cells that attaches to $\alpha$ on the right-hand side with both its $\alpha$-sides (if not, then the first block reattaches to the left side and after that every block starts attached on the right and then reattaches to the left, but that is not possible, since the last block has to reattach to the right of $\alpha$ ). This block constitutes a fold of width $\left\lfloor w^{\prime} / 2\right\rfloor \geq w /\left(2 d(12 p+13)^{2}\right)-1$.

It is easy to construct two reduced curves in the plane that intersect $\ell$ times and whose spirals and folds have depth and width at most $O(\sqrt{\ell})$, so Theorem 2.1 is essentially tight.

\section{The Torus Case}

Let $f_{n}$ be the $n$th Fibonacci numbers, with $f_{0}=0$ and $f_{1}=1$. Figure 2 shows a train track describing an arrangement of curves on the torus (using the usual planar representation of the torus identifying opposite sides of the square).

Fig. 2 A weighted train track on the torus (black lines)

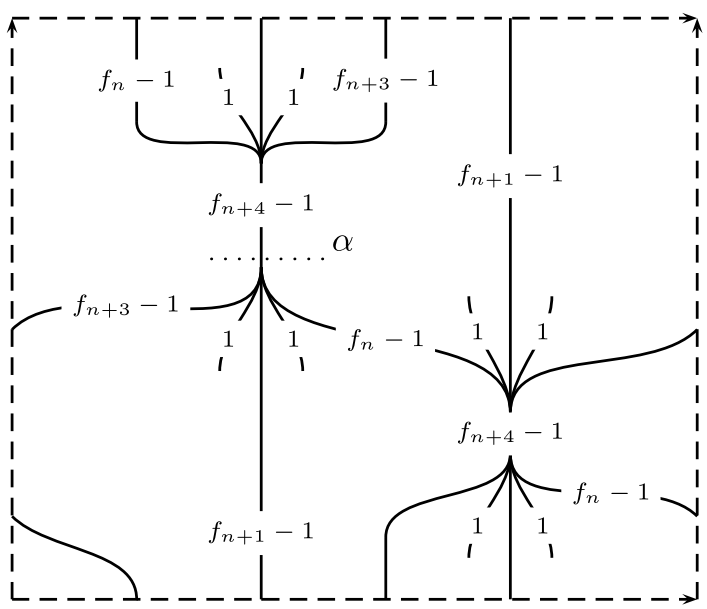


Without going into the formal details of weighted train tracks, let us describe the basic idea. Ignore, for the moment, the dotted line $\alpha$, it is not part of the train track. A line of weight $w$ in the diagram represents $w$ parallel curves. For example, in the upper left part of Fig. 2 we see five groups of parallel curves merge together. The leftmost group represents $f_{n}-1$ parallel curves, the third group $f_{n+1}-1$ (as we find out by tracing it across the dashed line), and the fifth group $f_{n+3}-1$ parallel curves. The second and fourth group represent the end of a single curve. These five groups merge, in a device called a switch, into a single group of $f_{n+4}-1=$ $\left(f_{n}-1\right)+1+\left(f_{n+1}-1\right)+1+\left(f_{n+3}-1\right)$ parallel curves, just to split up again into five groups of $f_{n+3}-1,1, f_{n+1}-1,1$ and $f_{n}-1$ parallel curves. Note that all lines in the diagram have a weight, and that when multiple lines merge (or split) in a switch, the weights add up consistently. Hence, if we replace each line with the number of parallel curves corresponding to its weight, we obtain a system of curves in the torus realizing the train track. We do not a priori know how the eight ends of the curves pair up and whether any closed curves are present, and, indeed, this depends on $n$. Clearly, there have to be at least four curves. We show that if $n \equiv 1 \bmod 3$, then the train track represents a system of exactly four curves and there are no closed curve components. Moreover, we argue that $\alpha$ (as shown in the picture) does not form a spiral, fold or lens with any of those four curves. Since one of the curves has to cross $\alpha$ at least $\left(f_{n+1}-1\right) / 4$ times, this will complete the argument.

To prove these results about the train track in Fig. 2, we change our point of view and reexpress the train track, or, more precisely, the connectivity of the curves represented by the train track, as a word equation. A word equation is an equation containing variables and letters from some alphabet, $\Sigma$. We use uppercase letters for variables and lowercase letters for elements of $\Sigma$. For example,

$$
a X=X a
$$

where $|X|=n$ is a simple word equation with given lengths, that is, the length of the word that a variable represents is specified. The equation is interpreted over the free monoid generated by $\Sigma$, so equality must hold letter by letter. In this particular example, the solution is $X=a^{n}$ (for any alphabet $\Sigma$ containing $a$ ). To take another example, consider

$$
a X_{0} X_{1}=X_{1} a X_{0}
$$

with $\left|X_{0}\right|=1, X_{1}=n$. Again, this equation is solvable, by $a X_{0} X_{1}=a^{n+2}$; however, the uniqueness of the solution depends on the parity of $n$. For even $n$, there is more than one solution, while for odd $n$ the solution is unique (assuming $\Sigma$ contains at least two letters, including $a$ ). Finally, the system

$$
a b X=X a b
$$

is solvable if and only if $|X|$ is even (in which case the solution is unique). We write $X[i]$ for the $i$ th letter in $X$ (for a particular solution), and $X[i: j]$ for the subword $X[i] X[i+1] \cdots X[j]$ of $X$.

Figure 3 shows the train track from Fig. 2 (no longer embedded) recast as a word equation. We have labeled four of the endpoints with $\{a, b, \bar{a}, \bar{b}\}$ and left the other four undetermined (the questions marks are unnamed, but distinct, variables). 


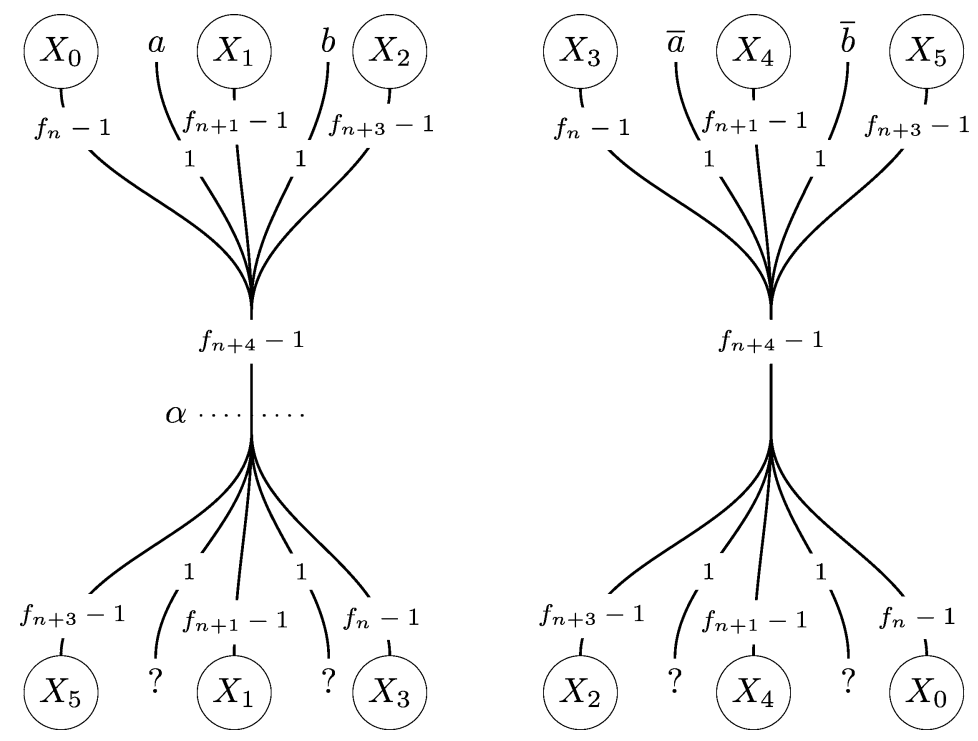

Fig. 3 Intersection of $\alpha$ with a system of curves containing no spirals

The word equation shown in Fig. 3 is

$$
X_{0} a X_{1} b X_{2} X_{3} \bar{a} X_{4} \bar{b} X_{5}=X_{5} ? X_{1} ? X_{3} X_{2} ? X_{4} ? X_{0}
$$

with given lengths $\left|X_{0}\right|=\left|X_{3}\right|=f_{n}-1,\left|X_{1}\right|=\left|X_{4}\right|=f_{n+1}-1,\left|X_{2}\right|=\left|X_{5}\right|=$ $f_{n+3}-1$ (the question marks have unit length).

Since the word equation is directly modeled on the connectivity of the train track, it is clear that any solution to (1) corresponds to a set of simple curves realizing the train track in Fig. $3 .^{3}$ This set includes four curves labeled $a, b, \bar{a}, \bar{b}$. Since there are only eight endpoints any other curve in this realization of the train track must be a closed curve. However, if there is a closed curve in the realization, we can relabel it in the solution to the word equation with an arbitrary letter. Hence, if we can show that the solution to (1) is unique, there cannot be any closed curves in the train track, and the realization consists of the four simple curves $a, b, \bar{a}, \bar{b}$. Indeed, in Lemmas 3.4 and 3.5 we show that (1) has a unique solution with the given length constraints as long as $n \equiv 1 \bmod 3$.

How do spirals translate into the world of words? Let $w \in \Sigma^{*}$ be the word we read along the curve $\alpha$ if we replace each intersecting curve with its letter. If any of the four curves $a, b, \bar{a}$ or $\bar{b}$, call it $\beta$, formed a spiral with $\alpha$, then there is an annulus $A$ containing at least three intersections of $\alpha$ with $\beta$. Consider a curve $\gamma$ in the train track which intersects $\alpha$ between two of these intersection points in $A$. The curve $\gamma$ does not intersect $\beta$ (the curves in the train track do not intersect) and it

\footnotetext{
${ }^{3}$ The reverse need not be true: by labeling the four ends on top of Fig. 3 by different letters, we exclude the case that any curve has both its endpoints there, a case that might very well occur for certain choices of $n$.
} 
cannot double back-forming a bigon-because there are no punctures in $A$ and we disallowed endpoints in an annulus witnessing a fold, and thus the bigon would be empty. Hence, $\gamma$ has to run parallel to $\beta$, implying that $w$ contains a word of the form $\beta x \beta x \beta$ (where $x$ is the word corresponding to the group of curves wedged between $\beta$, and $\beta$ is the letter representing the curve $\beta$ ) which contains the square $\beta x \beta x$. Hence, a spiral in the train track forces a square in the word along $\alpha$. In Lemma 3.5 we show that the word $X_{0} a X_{1} b X_{2}$ in the unique solution to (1) with the given lengths is square-free. Since $X_{0} a X_{1} b X_{2}$ records the intersections of the curves along $\alpha$, this implies that none of the curves forms a spiral with $\alpha$.

We finally observe that the arrangement of curves does not contain any folding: if we orient the curves in the train track, we see that they keep intersecting $\alpha$ in the same direction, whereas a fold requires a curve to intersect $\alpha$ in both directions (remember that a fold is contained in an annulus). Pending the proofs of Lemmas 3.4 and 3.5, we have thus established the following result.

Theorem 3.1 For any $n$ there are two simple curves $\alpha$ and $\beta$ on the torus that intersect at least $n$ times without forming either a lens, a fold or a spiral.

\subsection{Uniqueness}

Lemma 3.2 For $n \equiv 1(\bmod 3)$ a solution to the equation

$$
Y_{0} Y_{1}=Y_{1} Y_{0}
$$

with length constraints $\left|Y_{0}\right|=f_{n+2}$ and $\left|Y_{1}\right|=f_{n+1}+f_{n+3}$ over an arbitrary alphabet is uniquely determined by knowing $Y[i]$ for at least one odd and one even value of $i$.

Proof Let $\ell=\left|Y_{0} Y_{1}\right|=f_{n+1}+f_{n+2}+f_{n+3}=2 f_{n+3}$. Assume $Y=Y_{0} Y_{1}=Y_{1} Y_{0}$ is a solution to (2). By the equation, $Y[i]=Y\left[\left(i+f_{n+2}-1\right) \bmod \ell+1\right] .{ }^{4}$ The function $f(x)=\left(x+f_{n+2}-1\right) \bmod \ell+1$ splits the positions of $Y$ into $\operatorname{gcd}\left(f_{n+2}, \ell\right)$ orbits such that within each orbit the value of $Y$ is constant. For $n \equiv 1(\bmod 3), f_{n+2}$ is even, and, since $\operatorname{gcd}\left(f_{n+2}, f_{n+3}\right)=1, \operatorname{gcd}\left(f_{n+2}, \ell\right)=2$. Therefore, the function $f$ has exactly two orbits in this case: the even and the odd positions.

Lemma 3.3 For $n \equiv 1(\bmod 3)$ the equation

$$
X_{0} a X_{1} b X_{2}=X_{2} ? X_{1} ? X_{0}
$$

with length constraints $\left|X_{0}\right|=f_{n}-1,\left|X_{1}\right|=f_{n+1}-1$ and $\left|X_{2}\right|=f_{n+3}-1$ has a unique solution, and that solution is $(a b)^{\left(f_{n+4}-1\right) / 2}$.

Proof Consider the equation

$$
X=X_{0} a X_{1} a X_{2}=X_{2} ? X_{1} ? X_{0}
$$

\footnotetext{
${ }^{4}$ The $f_{n+2}$ nd position in $Y$ after $i$ (with wrap-around) is $\left(i+f_{n+2}-1\right) \bmod \ell+1$ since the first position in $Y$ is 1 .
} 
and let $X$ (and thereby $X_{0}, X_{1}, X_{2}$ ) be a solution over the alphabet $\{a, \diamond\}$ minimizing the number of occurrences of the letter $a$. Then (4) has a unique solution if and only if $X$ does not contain the letter $\diamond$. Counting occurrences of $a$ on both sides of $X_{0} a X_{1} a X_{2}=X_{2} ? X_{1} ? X_{0}$ it is clear that both ? must stand in for the letter $a$, so $X$ is a solution to the equation $X=X_{0} a X_{1} a X_{2}=X_{2} a X_{1} a X_{0}$. Adding $a X_{1} a$ in front of both sides of this equation shows that $X_{0}, X_{1}, X_{2}$ fulfill

$$
a X_{1} a X_{0} a X_{1} a X_{2}=a X_{1} a X_{2} a X_{1} a X_{0} .
$$

Now $Y_{0}=a X_{1} a X_{0}$ and $Y_{1}=a X_{1} a X_{2}$ form a solution to

$$
Y=Y_{0} Y_{1}=Y_{1} Y_{0}
$$

with length constraints $\left|Y_{0}\right|=f_{n+2}$ and $\left|Y_{1}\right|=f_{n+1}+f_{n+3}$. Moreover, $Y[1]=a$ and $Y\left[f_{n+1}+1\right]=a$, hence, noting that 1 is odd and $f_{n+1}+1$ is even, we can apply Lemma 3.2 to show that (6) and, thereby, (4), has a unique solution. This implies that

$$
X=X_{0} a X_{1} b X_{2}=X_{2} ? X_{1} ? X_{0}
$$

has a unique solution if it has any solution. However, it is easy to see that $X$ made up of alternating $a$ and $b$ is a solution, hence that solution is unique.

We think of the operator ${ }^{-}$as an involution on $\Sigma$, that is, $\overline{\bar{x}}=x$ for every $x \in \Sigma$. For example, $\overline{\bar{a}}=a$. We also let $\bar{\diamond}=\diamond$ and extend $\cdot$ to words in the natural way.

Lemma 3.4 Assume $n \equiv 1(\bmod 3)$. If the equation

$$
X=X_{0} a X_{1} b X_{2} X_{3} \bar{a} X_{4} \bar{b} X_{5}=X_{5} ? X_{1} ? X_{3} X_{2} ? X_{4} ? X_{0}
$$

with given lengths $\left|X_{0}\right|=\left|X_{3}\right|=f_{n}-1,\left|X_{1}\right|=\left|X_{4}\right|=f_{n+1}-1,\left|X_{2}\right|=\left|X_{5}\right|=$ $f_{n+3}-1$ has a solution, then that solution is unique and fulfills

$$
X_{0}=\bar{X}_{3}, X_{1}=\bar{X}_{4}, X_{2}=\bar{X}_{5}
$$

and

$$
X_{0} a X_{1} b X_{2}=\bar{X}_{2} ? X_{1} ? \bar{X}_{0}
$$

Proof Assume that $X$ is a solution of (8) over the alphabet $\{a, b, \bar{a}, \bar{b}, \diamond\}$ minimizing the number of letters in $\{a, b, \bar{a}, \bar{b}\}$. The solution is unique if and only if $X$ does not contain the character $\diamond$.

We claim that if $X=X_{0} a X_{1} b X_{2} X_{3} \bar{a} X_{4} \bar{b} X_{5}$ is a solution to (8) then so, by the symmetry of the equation, is

$$
X^{\prime}=\bar{X}_{3} a \bar{X}_{4} b \bar{X}_{5} \bar{X}_{0} \bar{a}_{1} \overline{b X}_{2}:
$$

namely, if $X$ fulfills (8) with the length constraints, then $X_{0} a X_{1} b X_{2}=X_{5}$ ? $X_{1}$ ? $X_{3}$ and $X_{3} \bar{a} X_{4} \bar{b} X_{5}=X_{2} ? X_{4} ? X_{0}$, so

$$
X_{3} \bar{a} X_{4} \bar{b} X_{5} X_{0} a X_{1} b X_{2}=X_{2} ? X_{4} ? X_{0} X_{5} ? X_{1} ? X_{3} .
$$


Now applying $\cdot$ to both sides establishes the claim.

Since $X^{\prime}$ has the same number of $\diamond$ characters as $X$, it is also a minimal solution. Moreover, $X^{\prime}$ and $X$ agree at the four positions in which a letter is specified by the equation. Therefore, $X$ and $X^{\prime}$ must equal each other. In other words, we have $X_{0}=\bar{X}_{3}, X_{1}=\bar{X}_{4}$ and $X_{2}=\bar{X}_{5}$. Then

$$
X_{0} a X_{1} b X_{2}=X_{5} ? X_{1} ? X_{3}=\bar{X}_{2} ? X_{1} ? \bar{X}_{0} .
$$

Now any solution of $X_{0} a X_{1} b X_{2}=\bar{X}_{2} ? X_{1} ? \bar{X}_{0}$ can be turned into a solution of $X_{0} a X_{1} b X_{2}=X_{2} ? X_{1}$ ? $X_{0}$ by replacing $\bar{a}$ with $a$ and $\bar{b}$ with $b$ (leaving any $\diamond$ letters untouched). By Lemma 3.3 the solution to $X_{0} a X_{1} b X_{2}=X_{2} ? X_{1}$ ? $X_{0}$ is unique and does not involve any $\diamond$ character. Consequently, the solution to $X_{0} a X_{1} b X_{2}=$ $\bar{X}_{2} ? X_{1} ? \bar{X}_{0}$ is unique and does not include a $\diamond$ character. But then the minimal solution $X$ of (8) does not contain a $\diamond$ character and is therefore the unique solution to that equation.

\subsection{Existence and Squares}

We have shown that a solution to (1) if it exists is unique. In this section we show that (1) with the given lengths has a solution and that this solution is square-free.

Lemma 3.5 Let $n \equiv 1(\bmod 3)$. Equation $(1)$ over $\{a, b, \bar{a}, \bar{b}\}$ with given lengths $\left|X_{0}\right|=\left|X_{3}\right|=f_{n}-1,\left|X_{1}\right|=\left|X_{4}\right|=f_{n+1}-1,\left|X_{2}\right|=\left|X_{5}\right|=f_{n+3}-1$ has a solution $X=X_{0} a X_{1} b X_{2} X_{3} \bar{a} X_{4} \bar{b} X_{5}$ and for that solution $X_{0} a X_{1} b X_{2}$ is square-free.

By Lemma 3.4 we know that the solution we construct will be the unique solution.

Proof of Lemma 3.5 Consider the equation

$$
Z=X_{0} a X_{1} b X_{2}=\bar{X}_{2} ? X_{1} ? \bar{X}_{0}
$$

with given lengths $\left|X_{0}\right|=f_{n}-1,\left|X_{1}\right|=f_{n+1}-1$, and $\left|X_{2}\right|=f_{n+3}-1$. Defining $X_{3}=\bar{X}_{0}, X_{4}=\bar{X}_{1}$ and $X_{5}=\bar{X}_{2}$ gives us $X_{0} a X_{1} b X_{2}=\bar{X}_{2} ? X_{1} ? \bar{X}_{0}=X_{5} ? X_{1} ? X_{3}$ and $X_{3} \bar{a} X_{4} \bar{b} X_{5}=\overline{X_{0} a X_{1} b X_{2}}={\overline{X_{2}}}_{2} ? X_{1} ? \bar{X}_{0}=X_{2} ? \bar{X}_{1} ? X_{0}=\bar{X}_{5} ? \bar{X}_{1} ? \bar{X}_{3}$, or, in other words, a solution to (1) with the given lengths. Hence to show that (1) has a solution, it is enough to show that (9) is solvable.

Let us write $Z_{v}^{u}$ for the result of replacing all occurrences of $u$ in $Z$ with $v$. We allow the specification of multiple replacements, for example, $Z_{a b}^{\bar{a} \bar{b}}$ is the result of replacing $\bar{a}$ with $a$ and $\bar{b}$ with $b$.

We split (9) into two equations. It is obvious that $Z$ is a solution to (9) if and only if $Z_{a b}^{\bar{a} \bar{b}}$ is a solution to

$$
Z^{\prime}=X_{0} a X_{1} b X_{2}=X_{2} ? X_{1} ? X_{0}
$$

over alphabet $\{a, b\}$ with given lengths $\left|X_{0}\right|=f_{n}-1,\left|X_{1}\right|=f_{n+1}-1,\left|X_{2}\right|=$ $f_{n+3}-1$, and $Z_{a \bar{a}}^{b \bar{b}}$ is a solution of

$$
Z^{\prime \prime}=X_{0} a X_{1} a X_{2}=\bar{X}_{2} ? X_{1} ? \bar{X}_{0}
$$


over alphabet $\{a, \bar{a}\}$ with given lengths $\left|X_{0}\right|=f_{n}-1,\left|X_{1}\right|=f_{n+1}-1,\left|X_{2}\right|=$ $f_{n+3}-1$.

In Lemma 3.3 we saw that (10) has the unique solution $Z^{\prime}=(a b)^{\left(f_{n+4}-1\right) / 2}$. Note that $Z^{\prime}$ does not contain any odd square, that is a square of the form $w w$ for which $|w|$ is odd. Therefore, $Z$ cannot contain any odd square. Hence, to conclude the argument, it is sufficient to show that (11) has a solution, and that this solution does not contain any even squares, that is a square of the form $w w$ for which $|w|$ is even. This proof uses a different approach and we leave it to Sect. 4: Lemma 4.9 shows that there is a solution to (11), and Lemma 4.6 together with Corollary 4.8 shows that this solution does not contain an even square.

\section{The Golden Ratio and Square-free Binary Words}

A square is a word of the form $w w$, where $w$ is not the empty word; we call the square even or odd, depending on the parity of $|w|$. A word is square-free if it does not contain a square as a subword. Any binary word of length at least 4 contains a square, while there are infinite words over a ternary alphabet which are square-free (discovered by Axel Thue [9, 10]).

This seems to close the case of binary words, but there are variations to be considered. For example, there are infinite binary words containing at most 3 squares, 00, 11 and $0101[4,5,7]$. In this section we show that there are infinite binary words that do not contain any even squares.

Theorem 4.1 There is an infinite binary word that does not contain an even square.

Traditionally, square-free words are constructed by repeatedly applying squarefree morphisms (morphisms that map square-free words to square-free words) to an initial square-free word. We proceed differently by showing that a particular sequence, namely the sequence $\mathbf{a}=\left(a_{n}\right)_{n \in \mathbb{N}}$ defined by

$$
a_{n}=\lfloor n \phi \bmod 2\rfloor=\lfloor n \phi\rfloor-2\lfloor n \phi / 2\rfloor
$$

does not contain an even square, where $\phi=(\sqrt{5}+1) / 2$ is the golden ratio. The sequence a is listed in Sloane's Encyclopedia of Integer Sequences [3, Sequence A085002]; it encodes the bits of $\phi: a_{2} \ell$ is the $\ell$ th binary bit of $\phi$.

\subsection{The Bits of the Golden Ratio}

Somewhat surprisingly, the bits of a can be generated by a simple recursive law which we exploit in the next section to show that a encodes a solution to (11). In this section we establish the necessary properties of a and moreover show that it does not contain any even squares.

We need two basic facts about the distribution of $n \phi \bmod 1$ and Fibonacci numbers. Let $\lfloor x\rceil$ be the integer nearest to $x$, and $\|x\|:=|x-\lfloor x\rceil|$ the distance of $x \in \mathbb{R}$ to its nearest integer. We will use the well-known identity $f_{k}=f_{k-1} \phi+\hat{\phi}^{k-1}$, where $\hat{\phi}=(1-\sqrt{5}) / 2$. 
Lemma 4.2 Let $k$ be an integer. If $0<\ell<f_{k}$, then $\left\|f_{k} \phi\right\|<\|\ell \phi\|$.

Proof Since $f_{k} / f_{k-1}$ is a continued fraction convergent of $\phi$, standard approximation results about continued fractions imply that $\left|f_{k-1} \phi-f_{k}\right| \leq\left|\ell \phi-\ell^{\prime}\right|$ for $0<\ell<f_{k}$ and any $\ell^{\prime}$; hence

$$
\min _{0<\ell<f_{k}}\|\ell \phi\|=\left\|f_{k-1} \phi\right\| \text {. }
$$

However, $\left\|f_{k-1} \phi\right\|=\left\|f_{k}-\hat{\phi}^{k-1}\right\|=\left\|-\hat{\phi}^{k-1}\right\|=\left|\hat{\phi}^{k-1}\right|>\left|\hat{\phi}^{k}\right|=\left\|f_{k+1}-\hat{\phi}^{k}\right\|=$ $\left\|f_{k} \phi\right\|$, and the lemma follows.

Lemma 4.3 Let $k \geq 2$ be an integer. Then

$$
\left\lfloor f_{k} \phi\right\rceil \bmod 2= \begin{cases}1 & \text { if } k \equiv 0,1(\bmod 3), \\ 0 & \text { if } k \equiv 2(\bmod 3) .\end{cases}
$$

Proof Since $\left|\hat{\phi}^{k}\right|<1 / 2$ for $k \geq 2$ we can conclude that $\left\lfloor f_{k} \phi\right\rceil=\left\lfloor f_{k+1}-\hat{\phi}^{k}\right\rceil=f_{k+1}$; since $f_{k+1}$ is even if and only if $k+1 \equiv 0(\bmod 3)$, the lemma is proved.

Lemma 4.4 Let $k, \ell \geq 1$ so that $k+\ell=f_{n}$ for some $n \geq 3$. Then

$$
a_{k}= \begin{cases}a_{\ell} & \text { if } n \equiv 0,1(\bmod 3) \\ 1-a_{\ell} & \text { if } n \equiv 2(\bmod 3)\end{cases}
$$

Lemma 4.4 implies that the last $f_{n-2}$ bits of $\mathbf{a}\left[1: f_{n-1}-1\right]$ are determined by $\mathbf{a}\left[1: f_{n-2}\right]$ in a very simple way that only depends on $n \bmod 3$. In the next section we investigate this recursive structure separately, and show that it satisfies (11).

Proof of Lemma 4.4 For the purposes of this proof, let $a_{m}=\lfloor m \phi \bmod 2\rfloor$, where $m \in \mathbb{Z}$; note that this extension of $\mathbf{a}$ is symmetric in the sense that it satisfies

$$
a_{m}=1-a_{-m}
$$

for all $m \neq 0$. Let $k, \ell$ and $n$ be as in the statement of the lemma. Define $\alpha=f_{n} \phi-$ $\left\lfloor f_{n} \phi\right\rceil$ and $\beta=\ell \phi-\lfloor\ell \phi\rfloor$. By Lemma 4.2 we have $|\alpha|<|\ell \phi-\lfloor\ell \phi\rceil| \leq \beta$. Now

$$
\begin{aligned}
a_{k} & =\left\lfloor\left(f_{n}-\ell\right) \phi \bmod 2\right\rfloor \\
& =\left\lfloor\left(\left\lfloor f_{n} \phi\right\rceil+\alpha-\lfloor\ell \phi\rfloor-\beta\right) \bmod 2\right\rfloor \\
& =\left\lfloor\left(\left\lfloor f_{n} \phi\right\rceil-\lfloor\ell \phi\rfloor-\beta\right) \bmod 2\right\rfloor \\
& =\left(\left\lfloor f_{n} \phi\right\rceil+\lfloor(-\lfloor\ell \phi\rfloor-\beta) \bmod 2\rfloor\right) \bmod 2 \\
& =\left(\left\lfloor f_{n} \phi\right\rceil+1-a_{\ell}\right) \quad \bmod 2 .
\end{aligned}
$$

In the third equality we used $|\alpha|<\beta$, in the fifth equality we used (14). The result now follows from Lemma 4.3.

We still need to show that the sequence a defined in (12) does not contain any even squares. For this we need the following results about multiples mod 1 of $\phi$. 
Lemma 4.5 Let $\ell$ be an integer. Let $m, m^{\prime}$ be such that $f_{m} \leq \ell<f_{m+1}$ and $f_{m^{\prime}}<$ $\ell \leq f_{m^{\prime}+1}$. Let

$$
S=\{0, \phi, 2 \phi, \ldots,(\ell-1) \phi\} \bmod 1
$$

be a set of multiples of $\phi$ modulo 1 (that is, on a circle of length 1).

(i) The longest segment non-intersecting $S$ has length $\phi^{-(m-2)}$.

(ii) The closest pair of points in $S$ is at distance $\phi^{-m^{\prime}}$.

Proof Both claims follow from the proof of the three-distance theorem by Sós; we used the version presented in [1] to calculate the three distances for our sequence. Claim (ii) also follows directly from Lemma 4.2 (if $i \phi \bmod 1$ and $j \phi \bmod 1$ are a closest pair, then so are 0 and $(i-j) \phi)$.

The following lemma proves Theorem 4.1.

Lemma 4.6 There are no $n, k \in \mathbb{N}$ so that $\boldsymbol{a}[n: n+2 k-1]=\boldsymbol{a}[n+2 k: n+4 k-1]$. In other words, a does not contain any even squares.

Proof Suppose that such $n$ and $k$ exist. Let $\Delta=2 k \phi \bmod 2$. Let $m^{\prime}$ be such that $f_{m^{\prime}}<k+1 \leq f_{m^{\prime}+1}$ and let $m$ be such that $f_{m} \leq 2 k<f_{m+1}$. Note $m>m^{\prime}$ and hence

$$
2 \phi^{-m^{\prime}}-\phi^{-(m-2)}=\phi^{-(m-2)}\left(2 \phi^{(m-2)-m^{\prime}}-1\right)>0 .
$$

By Lemma 4.5 (with $\ell=k+1$, using the lower bound on the distance of 0 and $\phi k \bmod 1)$ we have

$$
\phi k \bmod 1 \in\left[\phi^{-m^{\prime}}, 1-\phi^{-m^{\prime}}\right]
$$

and hence $\Delta \in\left[2 \phi^{-m^{\prime}}, 2-2 \phi^{-m^{\prime}}\right]$. Let $x=n \phi$ and let

$$
S=\{x, x+\phi, x+2 \phi, \ldots, x+(2 k-1) \phi\} \quad \bmod 1 .
$$

By Lemma 4.5 set $S$ contains a point $b_{0} \in\left[0, \phi^{-(m-2)}\right]$ and $b_{1} \in\left[1-\phi^{-(m-2)}, 1\right]$. Let $n_{0}, n_{1}$ be such that $b_{i}=\phi n_{i} \bmod 1($ for $i=0,1)$ and let $b_{i}^{\prime}=\phi n_{i} \bmod 2$ (for $i=0,1)$.

If $\Delta<1$ then

$$
2>\Delta+b_{1} \geq 1-\phi^{-(m-2)}+2 \phi^{-m^{\prime}}>1
$$

and hence $a_{n_{1}}=\left\lfloor b_{1}^{\prime} \bmod 2\right\rfloor \neq\left\lfloor b_{1}^{\prime}+\Delta \bmod 2\right\rfloor=a_{n_{1}+2 k}$.

If $\Delta>1$ then

$$
1<\Delta+b_{0} \leq 2-2 \phi^{m^{\prime}}+\phi^{-(m-2)}<2
$$

and hence $a_{n_{0}}=\left\lfloor b_{0}^{\prime} \bmod 2\right\rfloor \neq\left\lfloor b_{0}^{\prime}+\Delta \bmod 2\right\rfloor=a_{n_{0}+2 k}$.

Remark 4.7 As one of the referees pointed out it appears that the length of square words in a are all of the form $f_{3 k+2}$ for $k \in \mathbb{N}$. We verified this conjecture empirically for $\mathbf{a}\left[1: f_{20}-1\right]$ (using the recursive construction of a presented in the next section). 


\subsection{A Recursive Look at the Golden Ratio}

Let $w_{3}=1$ and

$$
w_{n}= \begin{cases}w_{n-1}\left(w_{n-1}\left[1: f_{n-2}\right]\right)^{R} & \text { if } n \equiv 0,1(\bmod 3) \\ w_{n-1}\left(\bar{w}_{n-1}\left[1: f_{n-2}\right]\right)^{R} & \text { if } n \equiv 2(\bmod 3)\end{cases}
$$

where $w^{R}$ denotes the reverse of $w$ and $\bar{w}$ the bitwise complement of $w$. The first few words are displayed in the following table.

\begin{tabular}{r|l|c}
\hline$n$ & $w_{n}$ & $\left|w_{n}\right|$ \\
\hline 3 & 1 & 1 \\
4 & 11 & 2 \\
5 & 1100 & 4 \\
6 & 1100011 & 7 \\
7 & 110001100011 & 12 \\
8 & 11000110001110011100 & 20 \\
9 & 110001100011100111001110001100011 & 33 \\
10 & 110001100011100111001110001100011100111001110001100011 & 54 \\
\hline
\end{tabular}

Note that $\left|w_{n}\right|=f_{n}-1$. By construction $w_{n-1}$ is a prefix of $w_{n}$, so it is meaningful to define $\mathbf{w}=\lim _{n \rightarrow \infty} w_{n}$. In Lemma 4.4 we showed that a satisfies the recursive construction law we used to define $\mathbf{w}$, so the following result does not come as a surprise.

\section{Corollary $4.8 w=a$.}

Proof We show that $\mathbf{w}\left[1: f_{n}-1\right]=\mathbf{a}\left[1: f_{n}-1\right]$ by induction on $n$. Equality holds in the base cases $n=3,4$. By Lemma 4.4, $\left(\mathbf{a}\left[f_{n-1}: f_{n}-1\right]\right)^{R}=\mathbf{a}\left[1: f_{n-2}\right]$ if $n \equiv$ $0,1(\bmod 3)$, and $\left(\mathbf{a}\left[f_{n-1}: f_{n}-1\right]\right)^{R}=\overline{\mathbf{a}}\left[1: f_{n-2}\right]$ for $n \equiv 2(\bmod 3)$. In either case, the last $f_{n-2}$ bits of a are determined by the first $f_{n-2}$ bits of a in exactly the same way that the last $f_{n-2}$ bits of $w_{n}$ are determined by the first $f_{n-2}$ bits of $w_{n}$ (using the recursive definition of $w_{n}$ ). Moreover, by inductive assumption, the first $f_{n-2}$ bits of $\mathbf{w}$ and $\mathbf{a}$ agree, completing the proof.

In particular, by combining Corollary 4.8 with Lemma 4.4 we get

$$
w_{n}= \begin{cases}w_{n}^{R} & \text { if } n \equiv 0,1(\bmod 3) \\ \bar{w}_{n}^{R} & \text { if } n \equiv 2(\bmod 3) .\end{cases}
$$

We still need to show that $w_{n}$ as defined above encodes a solution $Z^{\prime \prime}$ to (11)

$$
Z^{\prime \prime}=X_{0} a X_{1} a X_{2}=\bar{X}_{2} ? X_{1} ? \bar{X}_{0}
$$

with given lengths $\left|X_{0}\right|=f_{n}-1,\left|X_{1}\right|=f_{n+1}-1,\left|X_{2}\right|=f_{n+3}-1$. This is accomplished by the following lemma. 
Lemma 4.9 If $n \equiv 1(\bmod 3)$ then $w_{n+4}$ fulfills

$$
w_{n+4}=X_{0} S X_{1} S X_{2}=\bar{X}_{2} ? X_{1} ? \bar{X}_{0}
$$

over alphabet $\{0,1\}$ for some $X_{0}, X_{1}, X_{2}$ with given lengths $|S|=1$, and $\left|X_{0}\right|=$ $f_{n}-1,\left|X_{1}\right|=f_{n+1}-1,\left|X_{2}\right|=f_{n+3}-1$. In particular, (11) has the solution $Z^{\prime \prime}=$ $\left(w_{n+4}\right)_{a \bar{a}}^{S \bar{S}}$.

Proof Choose $X_{0}, X_{1}, X_{2}, Y_{0}, Y_{1}, Y_{2}$ over alphabet $\{0,1\}$ so that

$$
w_{n+4}=X_{0} S_{0} X_{1} S_{1} X_{2}=\bar{Y}_{2} ? Y_{1} ? \bar{Y}_{0}
$$

where $\left|S_{0}\right|=\left|S_{1}\right|=1$, and $\left|X_{0}\right|=\left|Y_{0}\right|=f_{n}-1,\left|X_{1}\right|=\left|Y_{1}\right|=f_{n+1}-1,\left|X_{2}\right|=$ $\left|Y_{2}\right|=f_{n+3}-1$. To establish the lemma, it is enough to show that $S_{0}=S_{1}$ and $X_{i}=Y_{i}$ for $1 \leq i \leq 3$.

Since $n \equiv 1(\bmod 3)$ by (16) we know that $w_{n+4}$ fulfills $w_{n+4}^{R}=\bar{w}_{n+4}$. Hence $\left(X_{0}\right)^{R}=Y_{0}$, and, since $X_{0}=w_{n+4}\left[1: f_{n}-1\right]=w_{n}$, we have $Y_{0}=\left(X_{0}\right)^{R}=\left(w_{n}\right)^{R}=$ $w_{n}=X_{0}$.

Similarly, $\left(X_{2}\right)^{R}=Y_{2}$, and, since $X_{2}=w_{n+4}\left[1: f_{n+3}-1\right]=w_{n+3}$, we have $Y_{2}=$ $\left(X_{2}\right)^{R}=\left(w_{n+3}\right)^{R}=w_{n+3}=X_{2}$.

Also, $\left(X_{1}\right)^{R}=\bar{Y}_{1}$ and $X_{1}=w_{n+4}\left[f_{n}+1: f_{n+2}-1\right]=w_{n+2}\left[f_{n}+1: f_{n+2}-1\right]=$ $\left(w_{n+2}\left[1: f_{n+1}-1\right]\right)^{R}=w_{n+1}^{R}$ (the second and the last equality hold because the $w_{k}$ are prefixes of each other and the third equality uses (16)); therefore $Y_{1}=\left(\bar{X}_{1}\right)^{R}=$ $\left(\left(\bar{w}_{n+1}\right)^{R}\right)^{R}=w_{n+1}^{R}=X_{1}$.

Finally, we have to show that the bits $S_{0}$ and $S_{1}$ in position $f_{n}$ and $f_{n+2}$ of $w_{n+4}$ are the same:

$$
\begin{aligned}
S_{0} & =w_{n+4}\left[f_{n+2}\right] \\
& =w_{n+3}\left[f_{n+2}\right]=w_{n+2}\left(w_{n+2}\left[1 . . f_{n+1}\right]\right)^{R}\left[f_{n+2}\right] \\
& =\left(w_{n+2}\left[1 . . f_{n+1}\right]\right)^{R}[1]=w_{n+2}\left[f_{n}\right] \\
& =w_{n+4}\left[f_{n}\right]=S_{1}
\end{aligned}
$$

which completes the argument.

Acknowledgements We would like to thank both referees for helpful and detailed comments that improved the presentation in both parts of the paper. We would also like to thank Jarek Grytczuk for prompting us to explore the connection to the golden ratio sequence.

\section{References}

1. Alessandri, P., Berthé, V.: Three distance theorems and combinatorics on words. Enseign. Math. (2) 44(1-2), 103-132 (1998)

2. Benzer, S.: On the topology of the genetic fine structure. Proc. Natl. Acad. Sci. 45, 1607-1620 (1959)

3. Cloitre, B.: Sequence A085002. http://www.research.att.com/ njas/sequences/A085002 (2003)

4. Entringer, R.C., Jackson, D.E., Schatz, J.A.: On nonrepetitive sequences. J. Comb. Theory Ser. A 16, 159-164 (1974) 
5. Fraenkel, A.S., Simpson, J.R.: How many squares must a binary sequence contain? Electron. J. Comb. 2 (1995)

6. Graham, R.L.: Problem 1. In: Open Problems at 5th Hungarian Colloquium on Combinatorics (1976)

7. Harju, T., Nowotka, D.: Binary words with few squares. Bull. Eur. Assoc. Theor. Comput. Sci. 89, 164 (2006)

8. Kratochvíl, J., Matoušek, J.: String graphs requiring exponential representations. J. Comb. Theory, Ser. B 53, 1-4 (1991)

9. Lothaire, M.: Combinatorics on Words. Cambridge Mathematical Library. Cambridge University Press, Cambridge (1997)

10. Lothaire, M.: Algebraic combinatorics on words. In: Encyclopedia of Mathematics and Its Applications, vol. 90. Cambridge University Press, Cambridge (2002)

11. Pach, J., Tóth, G.: Recognizing string graphs is decidable. Discrete Comput. Geom. 28(4), 593-606 (2002)

12. Schaefer, M., Štefankovič, D.: Decidability of string graphs. J. Comput. Syst. Sci. 68(2), 319-334 (2004)

13. Schaefer, M., Sedgwick, E., Štefankovič, D.: Algorithms for normal curves and surfaces. In: Computing and Combinatorics. Lecture Notes in Computer Science, vol. 2387, pp. 370-380. Springer, Berlin (2002)

14. Schaefer, M., Sedgwick, E., Štefankovič, D.: Recognizing string graphs in NP. J. Comput. Syst. Sci. 67(2), 365-380 (2003) (Special issue on STOC2002, Montreal, QC)

15. Schaefer, M., Sedgwick, E., Štefankovič, D.: Computing Dehn twists and geometric intersection numbers in polynomial time. Technical report TR05-009, DePaul University (2005)

16. Schaefer, M., Sedgwick, E., Štefankovič, D.: Spiraling and folding: the topological view. In: Bose, P. (ed.) Proceedings of the 19th Annual Canadian Conference on Computational Geometry, CCCG 2007, 20-22 August 2007, Carleton University, Ottawa, Canada, pp. 73-76 (2007) 